\title{
Variação diária da emissão de metano em solo cultivado com arroz irrigado no Sul do Brasil
}

\author{
Diurnal variation of methane emission from a paddy field under rice \\ cultivation in the Southern Brazil
}

\author{
Falberni de Souza Costa ${ }^{\text {I }}$ Cimélio BayerII Magda Aparecida de Lima ${ }^{\text {III }}$ \\ Rosa Toyoko Shiraishi Frighetto ${ }^{\mathrm{III}}$ Vera Regina Mussoi Macedo ${ }^{\mathrm{IV}}$ Elio Marcolin $^{\mathrm{IV}}$
}

- NOTA -

RESUMO

$\mathrm{O}$ metano $\left(\mathrm{CH}_{4}\right)$ apresenta um potencial de aquecimento 23 a 32 vezes maior do que o dióxido de $\mathrm{C}$ e a sua emissão diária em lavouras de arroz varia com a temperatura do solo e da água, e o metabolismo das plantas. O objetivo deste estudo foi determinar o horário apropriado à coleta de amostras de ar para quantificar a emissão média diária de $\mathrm{CH}_{4}$, informação fundamental para estudos visando a derivação de índices regionais de emissão desse gás. Três campanhas de monitoramento da emissão de $\mathrm{CH}_{4}$, com coletas em intervalos de 3 h, foram realizadas nos estágios de emissão da panícula (campanha 1) e de maturação de grãos (campanhas 2 e 3) do arroz sob diferentes sistemas de cultivo (convencional e plantio direto) na safra 2002/2003, na Estação Experimental do Instituto Rio Grandense do Arroz, em Cachoeirinha, RS. A coleta das amostras de ar foi realizada com seringas de poliestireno em câmaras de alumínio de $1 \mathrm{~m}^{2}$, sendo que as análises de metano foram realizadas por cromatografia gasosa. A emissão diária de $\mathrm{CH}_{4}$ apresentou padrão similar nas três campanhas de amostragem independente do sistema de cultivo. A faixa de emissão mínima (4-19mg $\mathrm{CH}_{4} \mathrm{~m}^{-2} \mathrm{~h}^{-1}$ ) ocorreu entre as 24 horas e o início da manhã seguinte (9 horas), e a faixa de emissão máxima (8-33mg $\left.\mathrm{CH}_{4} \mathrm{~m}^{-2} \mathrm{~h}^{-1}\right)$ do início à metade da tarde (12-15 horas). A emissão de $\mathrm{CH}_{4}$ foi relacionada à temperatura da água de inundação (campanhas 1 e 3) e do solo (campanha 2). Com base nas curvas de emissão diária e considerando aspectos operacionais como a viabilidade de análise cromatográfica das amostras no período de $24 \mathrm{~h}$ após a coleta, o intervalo entre 9 e 12 horas é recomendado para a avaliação das emissões de $\mathrm{CH}_{4}$ em lavouras de arroz irrigado quando objetiva-se a obtenção de valores equivalentes à emissão média diária desse gás.
Acredita-se que a alta relação entre a temperatura e a emissão de metano valida esse procedimento para as sub-regiões produtoras de arroz no Sul do Brasil (região litorânea e Sul do RS e região Sul de SC) pelo fato destas apresentarem comportamento diário similar de radiação solar e de temperatura do ar. A adequação do procedimento deve ser confirmada para as demais sub-regiões produtoras do Sul do Brasil, mas principalmente para outras regiões que apresentem condições ambientais diversas.

Palavras-chave: sistemas de cultivo, energia de ativação, metanogênese.

\section{ABSTRACT}

Methane $\left(\mathrm{CH}_{4}\right)$ has a global warming potential 23 to 32 times higher than carbon dioxide and its emission rate in rice fields should vary daily with water and soil temperature, and plant metabolism. This study aimed to identify the appropriate time interval for air sampling in order to quantify the mean daily $\mathrm{CH}_{4}$ emission rate, key information to future studies aiming the derivation of regional indexes of $\mathrm{CH}_{4}$ emission. Three campaigns were performed to evaluate diurnal variation in $\mathrm{CH}_{4}$ emissions (3h interval) from continuously flooded rice (Oryza sativa L. Cv. 'IRGA-422 CL') fields at different crop stages (panicle differentiation and ripening) and management systems (conventional tillage-CT and no-tillageNT) during 2002/2003 crop season in long-term experiment at the Instituto Rio Grandense do Arroz (IRGA), in Cachoeirinha, RS, Brazil. Static chamber method was used and the air samples collected with polystyrene syringes were analyzed by gas chromatography. Methane emission showed a same diurnal pattern in the three campaigns in the both tillage systems. The maximum range of emission (8-33mg $\mathrm{CH}_{4} m^{-2} h^{-1}$ ) was observed

'Universidade Federal do Acre (UFAC), Campus Floresta, Cruzeiro do Sul, AC, Brasil.

"Departamento de Solos, Universidade Federal do Rio Grande do Sul (UFRGS). Av. Bento Gonçalves, 7712, 91540-000, Porto

Alegre, RS, Brasil. E-mail: cimelio.bayer@ufrgs.br. Autor para correspondência.

IIIEmpresa Brasileira de Pesquisa Agropecuária (Embrapa), Jaguariúna, SP, Brasil.

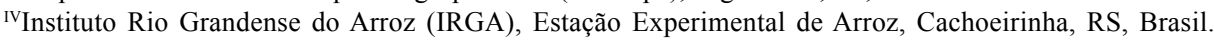


in the early afternoon (12.00 to 15.00 p.m.) followed by a decline to a minimum around midnight to next morning (4$\left.19 \mathrm{mg} \mathrm{CH}_{4} \mathrm{~m}^{-2} \mathrm{~h}^{-1}\right)$, in which fluxes were related to flood water temperature (campaign 1 and 3) or soil temperature (campaign 2). Taking into account the daily emission curves, plus operational aspects like chromatographic analysis of samples into the $24 \mathrm{~h}$ period after air sampling, the time interval from 9.00 to $12.00 \mathrm{a} . \mathrm{m}$. is recommended to studies aiming to evaluate mean daily $\mathrm{CH}_{4}$ emission in rice fields. The close relationship between temperature and methane emission allows us infer about the suitability of the gas sampling procedure for the different rice sub-regions of South of Brazil (littoral region and south of RS, and south of SC) that present the same daily behavior of solar radiation and air temperature. Even this, the appropriateness of this procedure should be tested for these different sub-regions of the South of Brazil but, mainly, for the world regions with diverse climatic conditions.

Key words: Management systems, activation energy, methanogenesis.

A variação diária na emissão de metano $\left(\mathrm{CH}_{4}\right)$ em solo cultivado com arroz irrigado por inundação tem relação com a mudança nas taxas metabólicas das plantas e dos microrganismos metanogênicos em resposta às mudanças na radiação solar e nas temperaturas do ar, da água de inundação e do solo no decorrer do dia (YANG \& CHANG, 1999). Além disso, a variação também é afetada pela fase de desenvolvimento do arroz devido à formação de aerênquimas, por onde $\mathrm{o} \mathrm{CH}_{4}$ difunde para a atmosfera (NOUCHI et al., 1990), e pela fonte de carbono disponível à metanogênese (WATANABE et al., 1999). Por sua vez, as práticas agrícolas de aração e gradagem do solo, aplicação de fertilizantes e a manutenção de uma lâmina de água sobre o solo aumentam a disponibilidade de $\mathrm{C}$ e ou a atividade dos microrganismos metanogênicos, aumentando a magnitude das emissões deste gás (CORTON et al., 2000).

A dependência do processo de emissão em relação à temperatura na emissão de metano em lavouras de arroz pode ser avaliada pela energia de ativação (Ea), a qual representa a quantidade mínima de energia que deve ser fornecida ao sistema para que haja emissão. A faixa geral da Ea é de 60 a $90 \mathrm{~kJ} \mathrm{~mol}^{-1}$, a qual equivale ao aumento de 2,5 a 3,5 vezes na produção de $\mathrm{CH}_{4}$ para cada aumento de $10^{\circ} \mathrm{C}(\mathrm{CONRAD}, 1989)$. Em condições de campo, a Ea pode ser maior visto que a temperatura pode afetar todos os processos relacionados à atividade das plantas, especialmente quanto à produção de exsudados orgânicos. Para CONRAD (1989), existe um balanço estreito entre o controle da temperatura e o controle do suprimento de substrato para a emissão de $\mathrm{CH}_{4}$.

A avaliação das emissões de $\mathrm{CH}_{4}$ em lavouras de arroz é tradicionalmente realizada pelo método da câmara fechada, sendo a análise das amostras de ar realizada por cromatografia gasosa. A representatividade dos resultados é fundamental à redução das incertezas em inventários, e pode ser relacionada à variação espacial e temporal (IPCC, 2001). Do ponto de vista de variação temporal, é importante a definição do horário do dia a ser realizada a coleta das amostras de ar de modo que o fluxo determinado seja equivalente ao fluxo médio diário de $\mathrm{CH}_{4}$, o que permite a realização de estimativas semanais, mensais e anuais (SASS \& FISHER, 1991). A partir dessas considerações, o objetivo deste estudo foi determinar o horário de coleta de amostras de ar que resulte em valor de emissão de $\mathrm{CH}_{4}$ equivalente à taxa média diária de emissão deste gás em lavoura de arroz irrigado no Sul do Brasil.

$\mathrm{O}$ estudo foi realizado em experimento instalado em 1994, na Estação Experimental do Instituto Rio Grandense do Arroz (IRGA), em Cachoeirinha (RS), em um Gleissolo Háplico Ta distrófico de textura franca (EMBRAPA, 1999). O clima da região é subtropical úmido e Cfa pela classificação de Köppen. O experimento consiste em dois sistemas de preparo de solo (preparo convencional-PC e plantio direto-PD), os quais foram dispostos em parcelas (28 x 40m) segundo o delineamento de blocos ao acaso, com três repetições. No presente estudo, apenas uma repetição foi avaliada. A cultivar "IRGA 422 CL" do arroz (Oryza sativa $\mathrm{L}$.) foi semeada mecanicamente e em linha (10/ 12/2002) e a inundação do solo ocorreu em 30/12/2002.

A campanha 1 (C1) de 24 horas foi realizada no solo em PC em 24-25/02/2003 (plantas em estágio de diferenciação das panículas); a campanha 2 (C2) no solo em PD em 17-18/03/2003; e a campanha 3 (C3) novamente no solo em PC, em 31/03-01/04/2003. Nestas duas últimas campanhas as plantas estavam no estágio de maturação de grãos. O método da câmara fechada foi utilizado para as amostragens de ar (MOSIER, 1989, IAEA, 1992), utilizando-se uma câmara de alumínio com $1 \mathrm{~m}^{2}$. Em cada intervalo de $3 \mathrm{~h}$, foi realizada a amostragem de ar da câmara que consistiu na coleta de amostras nos tempos $0,5,10,15,20$ e $25 \mathrm{~min}$, sendo que o tempo zero consistiu na amostragem do ar atmosférico feita a $2 \mathrm{~m}$ da superfície da água de inundação.

Seringas de poliestireno de $60 \mathrm{~mL}$ com bico tipo Luerlock de três vias foram utilizadas, e as temperaturas da água de inundação e do solo $(0,1 \mathrm{~m}$ de profundidade) foram registradas com auxilio de termômetro digital com haste metálica. A temperatura do ar atmosférico foi registrada na estação meteorológica do IRGA. As amostras de ar foram analisadas, em duplicata, por cromatografia gasosa, sendo que o desempenho do equipamento foi calibrado com padrão certificado de $5 \mu \mathrm{mol} \mathrm{mol}^{-1} \pm 9,54 \% \mathrm{de} \mathrm{CH}_{4} /$ 
ar sintético, com coluna megabore HP-A1/M (30m x $0,53 \mathrm{~mm} \times 15 \mu \mathrm{m})$ e detector de ionização de chama (IAEA, 1992), na Embrapa Meio Ambiente (Jaguariúna, SP), no prazo máximo de 24 horas após a coleta das amostras de ar. A relação entre as emissões de $\mathrm{CH}_{4}$ e a temperatura foi avaliada a partir da significância do coeficiente de determinação de equações polinomiais de $11^{\circ}$ grau em nível de $5 \%$. A equação de Arrhenius: $\mathrm{F}=\mathrm{ae}^{-\mathrm{Ea} / \mathrm{RT}}$ foi utilizada para cálculo da Energia de ativação (Ea) da emissão de $\mathrm{CH}_{4}$, em que F é a emissão de $\mathrm{CH}_{4}$, a é a constante de Arrhenius, e é logaritmo natural, Ea é a energia de ativação da emissão, R é a constante universal dos gases e $\mathrm{T}$ é a temperatura $(\mathrm{K})$ do meio considerado (SASS \& FISHER, 1991).

A concentração média de $\mathrm{CH}_{4}$ no ar atmosférico na $\mathrm{C} 1$ e na $\mathrm{C} 2$ foi três partes por milhão (ppm), enquanto que na $\mathrm{C} 3$ foi $2 \mathrm{ppm}$, com a média das campanhas aproximadamente $50 \%$ superior ao valor médio global (coletas em 40 locais de amostragem nos hemisférios Norte e Sul), de 1,77ppm (IPCC, 2007). Esses resultados se devem ao fato de que no cálculo da média global são incluídos somente resultados de locais remotos nos mares, sem influência continental, sobretudo de ar próximo a locais com lavouras de arroz irrigado ou sob influência urbana.

A forma da curva diária da emissão de $\mathrm{CH}_{4}$ foi muito similar nas três campanhas. A curva foi crescente no início, atingiu o valor máximo $(8-33 \mathrm{mg}$ $\left.\mathrm{CH}_{4} \mathrm{~m}^{-2} \mathrm{~h}^{-1}\right)$ entre 12 e 15 horas, decresceu, atingindo o valor mínimo (4-19 $\mathrm{mg} \mathrm{CH}_{4} \mathrm{~m}^{-2} \mathrm{~h}^{-1}$ ) na madrugada do dia seguinte (Figura 1a, b, c). A média diária da emissão de $\mathrm{CH}_{4}$ foi de $25( \pm 1,5), 6( \pm 0,3)$ e $11( \pm 1,8) \mathrm{mg} \mathrm{m}^{-2} \mathrm{~h}^{-1}$ nas campanhas $\mathrm{C} 1, \mathrm{C} 2$ e $\mathrm{C} 3$, respectivamente.

Diversas estratégias de amostragem podem ser definidas a partir da curva diária de emissão (Figura $1 \mathrm{a}, \mathrm{b}, \mathrm{c})$ visando obter valores equivalentes à emissão média diária de $\mathrm{CH}_{4}$. Entretanto, não há expectativa de que a definição de um horário determinado seja possível, devido à variabilidade das emissões em dias distintos no mesmo horário (Figura 1a, b, c), mas acredita-se que o mais adequado seja a definição de um intervalo de tempo para a amostragem. Dessa forma, considerando também aspectos operacionais, como a viabilidade de análise cromatográfica das amostras no período de $24 \mathrm{~h}$ após a coleta, a estratégia recomendada consiste na amostragem no período entre às 9 e às 12 horas.

Com base na emissão média de $\mathrm{CH}_{4}$ obtida a partir das coletas nesses dois horários ( 9 e 12h), obtevese valores de $25( \pm 1,7), 5( \pm 0,3)$ e $8( \pm 0,9) \mathrm{mg} \mathrm{CH}_{4} \mathrm{~m}^{-2} \mathrm{~h}^{-1}$ nas $\mathrm{C} 1, \mathrm{C} 2$ e $\mathrm{C} 3$, os quais foram similares e de mesma tendência que as taxas médias diárias estimadas (respectivamente 25, 6 e $11 \mathrm{mg} \mathrm{CH}_{4} \mathrm{~m}^{-2} \mathrm{~h}^{-1}$ ). Em média, os referidos valores são aproximadamente $10 \%$ inferiores às taxas médias diárias calculadas a partir da curva de emissão. Considerando-se a variabilidade intrínseca associada ao processo de geração e de emissão de $\mathrm{CH}_{4}$, e de amostragem dos GEE (IPCC, 2001, 2007), essa margem de erro pode ser considerada amplamente satisfatória, o que corrobora a adequação da adoção desse procedimento de avaliação no período entre 9 e $12 \mathrm{~h}$, quando tem-se por objetivo a estimativa das taxas médias diárias de $\mathrm{CH}_{4}$ em solo sob arroz irrigado, independente do sistema de cultivo.

A média do valor máximo (15h) nas duas amostragens no solo em PC foi de $28 \mathrm{mg} \mathrm{CH}_{4} \mathrm{~m}^{-2} \mathrm{~h}^{-1}$, enquanto no solo em PD este valor foi de $8 \mathrm{mg} \mathrm{CH}_{4} \mathrm{~m}^{-2}$ $\mathrm{h}^{-1}$. Da mesma forma, a média do valor mínimo nas duas amostragens no solo em PC foi de $13 \mathrm{mg} \mathrm{CH}_{4} \mathrm{~m}^{-2} \mathrm{~h}^{-1}$, enquanto no solo em PD foi de $4 \mathrm{mg} \mathrm{CH}_{4} \mathrm{~m}^{-2} \mathrm{~h}^{-1}$. Embora em fases diferentes de desenvolvimento do arroz, a amplitude dos valores estimados foi maior no solo em PC (15mg CH $\left.\mathrm{m}^{-2} \mathrm{~h}^{-1}\right)$ do que no solo em PD (4mg CH $\left.\mathrm{m}^{-2} \mathrm{~h}^{-1}\right)$. Esses resultados sugerem um possível potencial de mitigação das emissões de $\mathrm{CH}$ em lavouras de arroz irrigado sob PD, em comparação a lavouras em PC.

A emissão de $\mathrm{CH}_{4}$ foi positivamente relacionada com a temperatura da água de inundação (C1 e C3) e do solo (C2) (Figura 1d, e, f). Na C1 e na C3 houve incremento de $2,1(\mathrm{r}=0,80)$ e $5,7 \mathrm{mg} \mathrm{CH}_{4} \mathrm{~m}^{-2} \mathrm{~h}^{-1}$ $(\mathrm{r}=0,74)$, respectivamente, para cada grau de aumento na temperatura da água de inundação, enquanto que na $\mathrm{C} 2 \mathrm{o}$ incremento foi $1,2 \mathrm{mg} \mathrm{CH}_{4} \mathrm{~m}^{-2} \mathrm{~h}^{-1}(\mathrm{r}=0,70)$ para cada grau de aumento na temperatura do solo (Figura 1d, e, f). A Ea no solo em PC foi de 59 e $341 \mathrm{~kJ} \mathrm{~mol}^{-1}$ nas $\mathrm{C} 1$ e $\mathrm{C} 3$, respectivamente, enquanto que no solo em $\mathrm{PD}$ foi de $46 \mathrm{~kJ} \mathrm{~mol}^{-1}$ na C2. A Ea das $\mathrm{C} 1$ e C2 está na faixa citada por CONRAD (1989) $\left(60 \mathrm{a} 90 \mathrm{~kJ} \mathrm{~mol}^{-1}\right)$. Já o resultado da $\mathrm{C} 3$ foi quase quatro vezes maior do que o limite superior dessa faixa. Contudo, CONRAD (1989) ressalta que, em condições de campo, a Ea pode ser maior, visto que a temperatura pode afetar também a oxidação do $\mathrm{CH}_{4}$ e, adicionalmente, todos os processos relacionados à atividade das plantas, especialmente quanto à produção de exudatos orgânicos.

A maior média de emissão de $\mathrm{CH}_{4}$ no solo em $\mathrm{PC}$ na $\mathrm{C} 1$ em comparação à $\mathrm{C} 3$ pode estar relacionada à atividade metabólica mais intensa das plantas de arroz na diferenciação das panículas. Segundo WATANABE et al. (1999), nesta fase há a liberação de compostos orgânicos que podem servir de substrato aos microrganismos metanogênicos. $\mathrm{Na} \mathrm{C} 3$, com as plantas em maturação, a liberação desses compostos seria menor, em virtude da alocação de compostos orgânicos para o enchimento de grãos. Em adição, a temperatura 


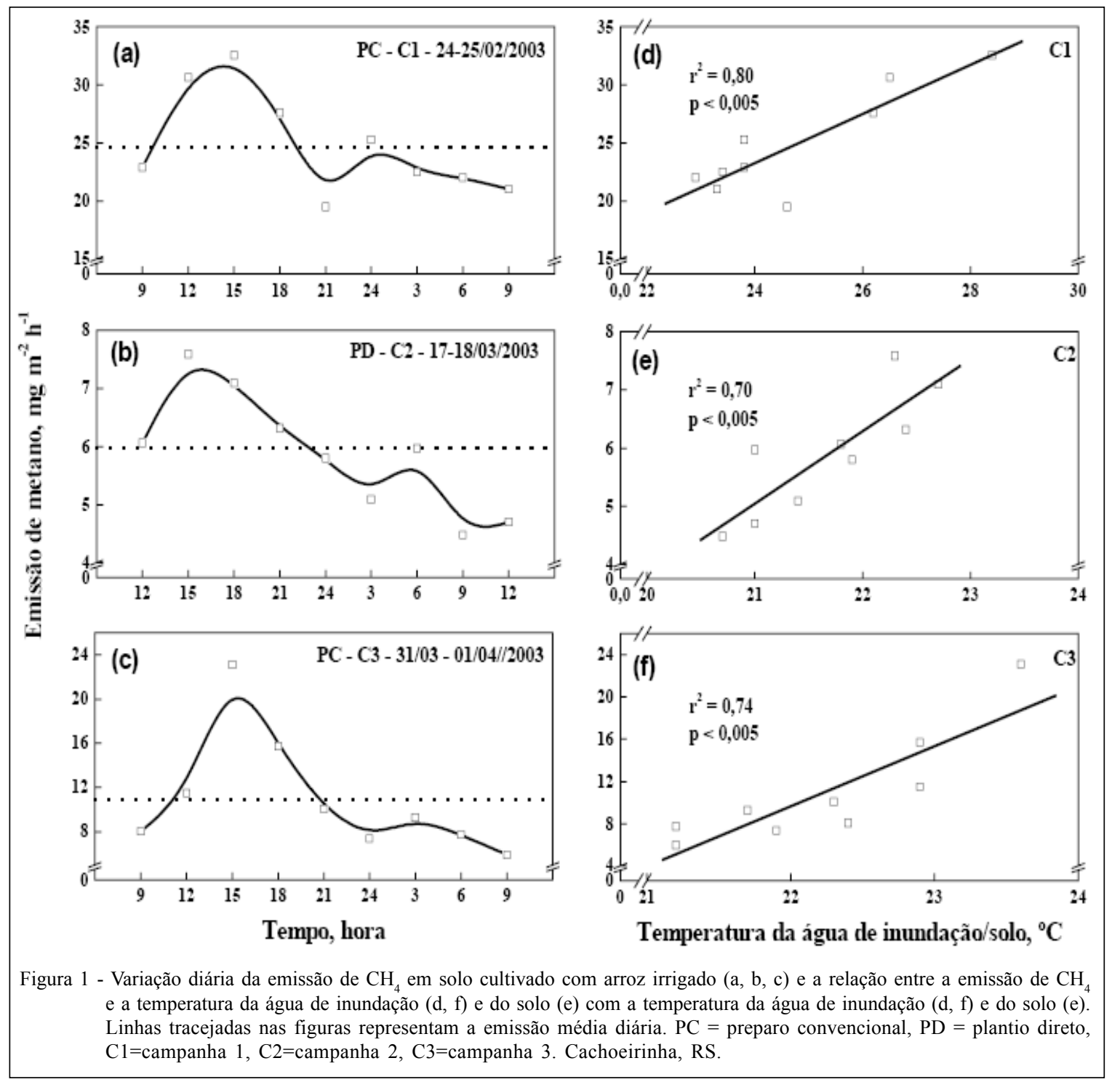

do ar e da água de inundação foi maior na $\mathrm{C} 1$ do que na C3. Estas condições podem estar somadas e traduzidas nos resultados da Ea para o PC. Na C1, com mais substratos e maior temperatura, a Ea foi 5,8 vezes menor do que na $\mathrm{C} 3$, com menos substratos e menor temperatura.

Com base nos resultados obtidos, concluise que a quantificação da emissão média diária de $\mathrm{CH}_{4}$ em solos cultivados com arroz pode ser realizada a partir da sua avaliação no intervalo entre 9 e $12 \mathrm{~h}$. Acredita-se que a alta relação entre a temperatura e a emissão de metano valide esse procedimento às sub-regiões produtoras de arroz no Sul do Brasil (região litorânea e sul do RS e região sul de SC) pelo fato destas apresentarem comportamento diário similar de radiação solar e de temperatura do ar. Ainda assim, a adequação desse procedimento deve ser validada para outras subregiões do Sul do Brasil, mas, principalmente, para regiões que apresentem condições ambientais diversas.

\section{AGRADECIMENTOS}

Os autores agradecem ao pesquisador Humberto Bohnen (Instituto Rio Grandense do Arroz), pelas sugestões no decorrer da pesquisa, e à Fundação de Amparo à Pesquisa do Estado do Rio Grande do Sul (FAPERGS), ao Conselho Nacional de Desenvolvimento Científico e Tecnológico (CNPq) e ao Ministério da Ciência e Tecnologia (MCT), pelo apoio financeiro ao Projeto Pronex 04/850.0 e Rede Agrogases.

\section{REFERÊNCIAS}

CONRAD, R. Control of methane production in terrestrial ecosystems. In: ANDREAE, M.O.; SCHIMEL, D.S. Exchange of traces gases between terrestrial ecosystems and the

Ciência Rural, v.38, n.7, out, 2008. 
atmosphere: report of the Dahlem Workshop. Berlin: John Wiley \& Sons, 1989. p.39-58.

CORTON, T.M. et al. Methane emission from irrigated and intensively managed rice field in Central Luzon (Philippines) Nutrient Cycling in Agroecosystems, v.58, p.37-53, 2000.

EMPRESA BRASILEIRA DE PESQUISA AGROPECUÁRIAEMBRAPA. Centro Nacional de Pesquisa de Solos. Sistema Brasileiro de Classificação de Solos. Brasília: Embrapa Produção de Informação, 1999. 412p.

IAEA. International Atomic Energy Agency. Manual on measurement of methane and nitrous oxide emissions from agriculture. Vienna:IAEA, 1992. 91p. (IAEA TECDOC, 674).

IPCC. Intergovernmental Panel on Climate Change. Climate change 2001: the scientific basis. United Kingdom: Cambridge University, 2001. 881p

IPCC. Intergovernmental Panel on Climate Change. Climate Change 2007: the physical science basis. United Kingdom and New York, USA: Cambridge University, 2007. 996p.
MOSIER, A.R. Chamber and isotopic techniques. In: ANDREAE, M.O.; SCHIMEL, D.S. Exchange of traces gases between terrestrial ecosystems and the atmosphere: report of the Dahlem Workshop. Berlin: John Wiley \& Sons, 1989. p.175-187

NOUCHI, I. et al. Mechanisms of methane transport from the rhizosphere to the atmosphere through rice plants. Plant Physiology, v.94, n.1, p.59-66, 1990.

SASS, R.L.; FISHER, F.M. Methane emission from rice field as influenced by solar radiation, temperature, and straw incorporation. Global Biogeochemical Cycles, v.5, p.335-350, 1991 .

WATANABE, A. et al. Evaluations of origins of $\mathrm{CH}_{4}$ carbon emitted from rice paddies. J ournal of Geophysical Research, v.104, n.D19, p.23623-23629, 1999.

YANG, S.-S.; CHANG, H.-L. Diurnal variation of methane emission from paddy fields at different growth stages of rice cultivation in Taiwan. Agriculture, Ecosystems \& Environment, v.76, p.75-84, 1999. 